\title{
Missouri Emergency Department Visits for Carbon Monoxide Poisoning
}

\author{
Fei Wu* and Carol Braun \\ Missouri Department of Health and Senior Services, Jefferson City, MO, USA
}

\section{Objective}

To obtain accurate estimates of the carbon monoxide $(\mathrm{CO})$ poisoning burden and guide prevention efforts. This study employs the current Missouri CO poisoning surveillance systems and describes the recent status of emergency department (ED) visits due to $\mathrm{CO}$ poisoning in Missouri.

\section{Introduction}

$\mathrm{CO}$ poisoning poses a significant public health burden. It is preventable, yet it remains a leading cause of poisoning in the United States. An effective surveillance system is very important for targeting and monitoring $\mathrm{CO}$ poisoning.

\section{Methods}

ED data was analyzed from the Missouri Electronic Surveillance System for the Early Notification of Community-based Epidemics (ESSENCE). The ED chief complaints of CO poisoning in ESSENCE contain keywords such as "carbon" or "monoxide". The Missouri Health Strategic Architectures and Information Cooperative (MOHSAIC) database was used to collect the other information about the CO poisoning cases. Statistical Analysis Software (SAS) (version 9.3) was applied for all the analyses in this study.

\section{Results}

This study was performed to estimate the ED visits from January 1, 2012 through June 30, 2013 in Missouri from all-causes (work-related, non-work-related, intentional, unintentional, fire, non-fire and unknown) of CO poisoning. There were $119 \mathrm{ED}$ visits for confirmed cases of $\mathrm{CO}$ poisoning in Missouri during this time period. Most ED visits for $\mathrm{CO}$ poisoning were among adults $(70.6 \%, 18-65$ year-old) and whites $(64.7 \%)$. No significant difference was observed between males and females. More poisonings occurred at the beginning of the winter months and the end of the winter months, which were October and February, respectively. St. Louis County, St. Louis City and Jackson County (includes large portion of Kansas City) had the highest numbers of $\mathrm{CO}$ poisoning during the study time period. All the ED visits in this study had carboxyhemoglobin $(\mathrm{COHb})$ levels available, the $50 \%$ median of the $\mathrm{COHb}$ tests was $3.0 \%$.

\section{Conclusions}

This study demonstrated the utility and importance of ED data as a surveillance tool as cases of $\mathrm{CO}$ poisoning were identified in ESSENCE that were not identified in the passive surveillance system (MOHSAIC). The study also demonstrated the potential of ED data to assist in developing effective, more targeted prevention strategies for $\mathrm{CO}$ poisoning.

\section{Keywords}

Carbon Monoxide Poisoning; Emergency Department visits; ESSENCE

\section{Acknowledgments}

Thank you to Eden Dietle, Linton Bartlett, Lori Harris and Amy Forbis at the Missouri Department of Health and Senior Services for reviewing this abstract.

*Fei Wu

E-mail: Fei.Wu@health.mo.gov 